Homology, Homotopy and Applications, vol.8(2), 2006, pp.133-155

\title{
AN EXPLICIT CLASSIFICATION OF THREE-STAGE POSTNIKOV TOWERS
}

\author{
PETER I. BOOTH
}

(communicated by R. Brown)

\begin{abstract}
The problems of classifying Hurewicz fibrations whose fibres have just two non-zero homotopy groups and classifying 3-stage Postnikov towers are substantially equivalent.

We investigate the case where the fibres have the homotopy type of $K(G, m) \times K(H, n)$, for $1<m<n$. Our solution uses a classifying space $M_{\infty}$, i.e. a mapping space whose underlying set consists of all null homotopic maps from individual fibres of the path fibration $P K(G, m+1) \rightarrow K(G, m+1)$ to the space $K(H, n+1)$, and the group $\mathcal{E}(K(G, m) \times K(H, n))$ of based homotopy classes of based self-homotopy equivalences of $K(G, m) \times K(H, n)$. If $B$ is a given space, then a group action

$$
\mathcal{E}(K(G, m) \times K(H, n)) \times\left[B, M_{\infty}\right]^{0} \rightarrow\left[B, M_{\infty}\right]^{0}
$$

is defined, and the orbit set $\left[B, M_{\infty}\right]^{0} / \mathcal{E}(K(G, m) \times K(H, n))$ is shown to classify the above fibrations over $B$ up to fibrewise homotopy type.

Our explicit definitions of the classifying spaces, together with our computationally effective group actions, are advantageous for computations and further developments. Two stable range simplifications are given here, together with a classification result for cases where $B$ is a product of spheres.
\end{abstract}

Dedicated to L. Gaunce Lewis, Jr (1949-2006)

The author would like to express his appreciation of the advice, concerning this paper, that was given by Gaunce Lewis. It included detailed comments concerning the presentation and restructuring of the material, and a helpful suggestion concerning future developments. This was done at a time when Gaunce was well aware of the seriousness and immediacy of the health problems that he faced. It is but one example of his helpfulness, kindness and generosity.

Received July 20, 2005, revised August 22, 2006; published on September 27, 2006.

2000 Mathematics Subject Classification: 55R15, 55R35, 55S45, 55P20.

Key words and phrases: Fibration, Postnikov System, classifying space, Eilenberg-MacLane space.

Copyright (c) 2006, International Press. Permission to copy for private use granted. 
Homology, Homotopy and Applications, vol. 8(2), 2006

\section{Main Results}

Let $F$ and $B$ be spaces. Then $\mathrm{FHT}(F: B)$ will denote the set of fibrewise homotopy types (= fibre homotopy types) of Hurewicz fibrations over $B$ with fibres homotopy equivalent to $F$.

If $K(G, m)$ is an Eilenberg-MacLane space with $m>0$, and $B$ is a space, then there is an obvious group action

$$
\text { Aut } G \times[B, K(G, m+1)]^{0} \rightarrow[B, K(G, m+1)]^{0},
$$

where the $[-,-]^{0}$ notation refers to based homotopy classes of based maps. It has long been known that if $B$ has the homotopy type of a simply connected $C W$ complex, then $\operatorname{FHT}(K(G, m): B)$ is determined — via a canonical bijection — by the orbit set

$$
[B, K(G, m+1)]^{0} / \text { Aut } G \approx H^{m+1}(B ; G) / \text { Aut } G \text {. }
$$

(see Section 23 of [St] for an early version of the result, and 5.2.2 of [Ba] for a proof).

In this paper, we generalize that theorem to the case where the fibre is a product of two Eilenberg-MacLane spaces. Our solution uses the classifying space $M_{\infty}$, introduced on p.89 of [B1], with underlying set consisting of all null homotopic maps from individual fibres of the path fibration $p: P K(G, m+1) \rightarrow K(G, m+1)$ [S, Cor.2.8.8] to the space $K(H, n+1)$. More details are given in Section 4 .

If $X$ and $Y$ are spaces and $y \in Y$, then $c(y)$ will be used to denote the constant map from $X$ to $Y$ with value $y$. In particular, the basepoint of $M_{\infty}$ will be the constant map $c(e): \Omega K(G, m+1) \rightarrow K(H, n+1)$, where $\Omega$ indicates a loop space and $e$ is the identity of $K(H, n+1)$.

Let $X$ be a based space. Then $\mathcal{E}(X)$ will denote the group of based homotopy classes of based self-homotopy equivalences of $X$, with the operation of composition.

The group $\mathcal{E}(K(G, m) \times K(H, n))$ can be identified with the collection of all $2 \times 2$ matrices of the form

$$
\left(\begin{array}{cc}
\theta & 0 \\
{[\psi]} & \phi
\end{array}\right),
$$

where $\theta \in$ Aut $G, \phi \in$ Aut $H, \psi$ is a based map from $K(G, m)$ to $K(H, n)$, and $[\psi]$ the corresponding homotopy class in $[K(G, m), K(H, n)]^{0}$ (see [R, Example 4.1]). Multiplication within this group agrees with matrix multiplication and composition of terms, in the expected sense (see also (2.7)).

If $q: Y \rightarrow B$ is a fibration and $b \in B$, then we will use $Y \mid b$ to denote the fibre of $q$ over $b$.

A pointed space will be said to be well pointed if the inclusion of the basepoint in the space satifies the homotopy extension property.

Proposition 1.1 (The Basic Group Action). Let $m$ and $n$ be integers such that $0<m<n, G$ and $H$ be Abelian groups, and $B$ be a well pointed space. Then there 
is a group action

$$
\mathcal{E}(K(G, m) \times K(H, n)) \times\left[B, M_{\infty}\right]^{0} \rightarrow\left[B, M_{\infty}\right]^{0}
$$

defined by the following procedure.

Let $k: B \rightarrow M_{\infty}$ be a based map, and the map $p_{\infty}: M_{\infty} \rightarrow K(G, m+1)$ the obvious projection (see (4.1)). Then there is a useful based map $k_{1}=p_{\infty} \circ k: B \rightarrow$ $K(G, m+1)$. We note that if $b \in B$, then $k(b)$ maps $P K(G, m+1) \mid k_{1}(b)$ to $K(H, n+$ 1).

The automorphisms $\theta \in A u t(G)$ and $\phi \in A u t(H)$ induce associated homeomorphisms $\underline{\theta}: K(G, m+1) \rightarrow K(G, m+1)$ and $\underline{\phi}: K(H, n+1) \rightarrow K(H, n+1)$, respectively. Then $\underline{\theta}$ induces a homeomorphism

$$
\theta_{x}: P K(G, m+1)|x \rightarrow P K(G, m+1)| \underline{\theta}(x), \quad \theta_{x}(y)=\underline{\theta} \circ y,
$$

where $x \in K(G, m+1)$ and $y \in P K(G, m+1) \mid x$. The map

$$
\underline{\phi} \circ k(b) \circ \theta_{k_{1}(b)}^{-1}: P K(G, m+1) \mid \underline{\theta}\left(k_{1}(b)\right) \rightarrow K(H, n+1)
$$

is an element of $M_{\infty}$.

We then have maps

$$
B \times\{0\} \rightarrow M_{\infty}, \quad(b, 0) \mapsto \underline{\phi} \circ k(b) \circ \theta_{k_{1}(b)}^{-1},
$$

where $b \in B$, and

$$
\left\{b_{0}\right\} \times I \rightarrow M_{\infty},\left(b_{0}, t\right) \mapsto\left(y \mapsto\left(\psi\left(\underline{\theta}^{-1} \circ y\right)\right)(t)\right),
$$

where $b_{0}$ is the basepoint of $B, t \in I, y \in K(G, m)=\Omega K(G, m+1) \subset P K(G, m+$ $1)$ is viewed as a loop in $K(G, m+1)$, and $\psi$ is a based map $K(G, m) \rightarrow K(H, n)$. We notice that these two maps into $M_{\infty}$ agree at $\left(b_{0}, 0\right)$, i.e. both take that point to the constant map $c(e): \Omega K(G, m+1) \rightarrow K(H, n+1)$.

A single application of the homotopy extension property then determines - up to based homotopy class - a based map

$$
\left(\begin{array}{cc}
\theta & 0 \\
\psi & \phi
\end{array}\right) \cdot k: B=B \times\{1\} \rightarrow M_{\infty} .
$$

The required group action is then induced by the rule

$$
\left(\left(\begin{array}{cc}
\theta & 0 \\
\psi & \phi
\end{array}\right), k\right) \mapsto\left(\begin{array}{cc}
\theta & 0 \\
\psi & \phi
\end{array}\right) \cdot k
$$

We define the three-stage Postnikov tower over $B, \tau\left(k_{1}, k_{2}\right)$, to consist of the principal fibrations $p_{1}: E_{1} \rightarrow B$ and $p_{2}: E_{2} \rightarrow E_{1}$, determined by the $k$-invariants $k_{1}$ and $k_{2}$, respectively.

We define $t\left(k_{1}, k_{2}\right)$, the Postnikov fibration over $B$ asssociated with $\tau\left(k_{1}, k_{2}\right)$, to be the fibration $p_{1} \circ p_{2}$

The based map $k: B \rightarrow M_{\infty}$ determines a Postnikov fibration $t\left(k_{1}, k_{2}\right)$ over $B$, where the first $k$-invariant is $k_{1}$, as described in Proposition 1.1, and the second 
$k$-invariant $k_{2}$ is specified by the rule

$$
(b, y) \mapsto k(b)(y),
$$

where $k_{1}(b)=p(y)$. Thus $k_{2}$ denotes a map from the second stage of the tower, i.e. the pullback space $B \sqcap P K(G, m+1)$ determined by $k_{1}$ and $p$, to the space $K(H, n+1)$. Further details concerning $k$ and $t\left(k_{1}, k_{2}\right)$ are given in (4.2).

Now $k$ is basepoint preserving, so $k_{2} \mid\left(\left\{b_{0}\right\} \times \Omega K(G, m+1)\right)$ is the constant map with value the identity $e$ of $K(H, n+1)$. It follows that the fibration $t\left(k_{1}, k_{2}\right)$ has distinguished fibre $K(G, m) \times K(H, n)$ (see $(2.7 .7)$ of [B1]).

Theorem 1.2 (The Basic Classification Theorem). Let $m$ and $n$ be integers such that $1<m<n$, and $G$ and $H$ be Abelian groups. Further the well pointed space $B$ has the homotopy type of a simply connected $C W$-complex, so $B$ could be any simply connected $C W$-complex.

Then there is a canonical bijection:

$$
\left[B, M_{\infty}\right]^{0} / \mathcal{E}(K(G, m) \times K(H, n)) \rightarrow \mathrm{FHT}(K(G, m) \times K(H, n): B)
$$

determined by the rule $[[k]] \rightarrow\left[t\left(k_{1}, k_{2}\right)\right]$. Thus the orbit $[[k]]$ of the based homotopy class $[k]$ of the based map $k: B \rightarrow M_{\infty}$ corresponds to the fibrewise homotopy type $\left[t\left(k_{1}, k_{2}\right)\right]$ of the Postnikov fibration $t\left(k_{1}, k_{2}\right)$ over $B$ with distinguished fibre $\bar{K} \times \bar{L}$. The bijection is natural relative to $B$.

We recall that if $p: X \rightarrow B$ is a fibration with fibre of the homotopy type of $K(G, m) \times K(H, n)$, then $p$ has the fibrewise homotopy type of an associated Postnikov fibration over $B$. Hence $F H T(\bar{K} \times \bar{L}: B)$ can be interpreted either as previously, or as the set of FHTs of Postnikov fibrations over $B$ with distinguished fibre $K(G, m) \times K(H, n)$.

We now give a handier version of this theorem, at least for stable range situations. The essential difference is that the previous group action was defined using composition of functions and the often harder to compute homotopy extension property, whereas, in the following case, the action is more conveniently defined using composition and pointwise addition of functions only.

Proposition 1.3 (The Second Group Action). Let $m$ and $n$ be integers such that $0<m<n$, and $G$ and $H$ be Abelian groups. Then there is an explicit group action

$$
\mathcal{E}(K(G, m+1) \times K(H, n+1)) \times\left[B, M_{\infty}\right]^{0} \rightarrow\left[B, M_{\infty}\right]^{0}
$$

induced by the rule

$$
\left(\left(\begin{array}{cc}
\theta & 0 \\
\chi & \phi
\end{array}\right), k\right) \mapsto\left(b \mapsto \underline{\phi} \circ k(b) \circ \theta_{k_{1}(b)}^{-1}+c\left(\chi k_{1}(b)\right)\right),
$$

where $\theta \in$ Aut $G, \phi \in$ Aut $H, \chi: K(G, m+1) \rightarrow K(H, n+1)$ and $k: B \rightarrow M_{\infty}$ are based maps, and $b \in B$. We note that the constant map $c\left(\chi k_{1}(b)\right)$, the composite $\phi \circ$ $k(b) \circ \theta_{k_{1}(b)}^{-1}$ and their sum are all maps from $P K(G, m+1) \mid \underline{\theta}\left(k_{1}(b)\right)$ to $K(H, n+\overline{1})$.

We will show in Section 6 that, under stable range conditions, this action is equivalent to that of Proposition 1.1, leading to the following result. 
Theorem 1.4 (A Stable Range Classification Theorem). Let $m$ and $n$ be integers such that $1<m<n<2 m, G$ and $H$ be Abelian groups, and the well pointed space $B$ have the homotopy type of a simply connected $C W$-complex. Then there is a canonical bijection

$$
\left[B, M_{\infty}\right]^{0} / \mathcal{E}(K(G, m+1) \times K(H, n+1)) \rightarrow \operatorname{FHT}(K(G, m) \times K(H, n): B)
$$

determined by the rule $[[k]] \mapsto\left[t\left(k_{1}, k_{2}\right)\right]$. Thus the based map $k: B \rightarrow M_{\infty}$ is the classifying map for the Postnikov fibration $t\left(k_{1}, k_{2}\right)$ over $B$ with distinguished fibre $\bar{K} \times \bar{L},[k]$ is the homotopy class of $k,[[k]]$ the orbit of that homotopy class, and $\left[t\left(k_{1}, k_{2}\right)\right]$ the fibrewise homotopy type of $t\left(k_{1}, k_{2}\right)$. The bijection is natural relative to $B$.

Let $\theta_{*}$ and $\phi_{*}$ be the automorphisms of $H^{m+1}(B ; G)$ and $H^{n+1}(B ; H)$ that are induced by the automorphisms $\theta$ and $\phi$ of their coefficient groups $G$ and $H$, respectively, and $\chi_{*}: H^{m+1}(B ; G) \rightarrow H^{n+1}(B ; H)$ be induced by the cohomology operation $[\chi] \in H^{n+1}(K(G, m+1) ; H)$.

Proposition 1.5 (The Third Group Action). Let $m$ and $n$ be integers such that $0<m<n$, and $G$ and $H$ be Abelian groups. Then there is a group action

$$
\begin{aligned}
\mathcal{E}(K(G, m+1) & \times K(H, n+1)) \times\left(H^{m+1}(B ; G) \oplus H^{n+1}(B ; H)\right) \\
& \rightarrow\left(H^{m+1}(B ; G) \oplus H^{n+1}(B ; H)\right),
\end{aligned}
$$

determined by the rule

$$
(\langle\theta,[\chi], \phi\rangle, \kappa, \lambda) \mapsto\left(\theta_{*}(\kappa), \phi_{*}(\lambda)+\chi_{*}(\kappa)\right),
$$

where $\kappa \in H^{m+1}(B ; G)$ and $\lambda \in H^{n+1}(B, H)$.

Theorem 1.6 (Another Stable Range Classification Theorem). Let $G$ and $H$ be Abelian groups, $m$ and $n$ be integers, and the well pointed space $B$ have the homotopy type of a $C W$-complex. We will assume that $1<m<n<2 m$, and that the connectivity of $B$ is greater than $n-m+1$.

(i) The stable range assumptions ensure that the fibrations in question have the fibrewise homotopy types of principal $K(G, m) \times K(H, n)$-fibrations. So, in this particular situation, $\mathrm{FHT}(K(G, m) \times K(H, n): B)$ can be interpreted in three ways, i.e. as the set of fibrewise homotopy types of either Hurewicz fibrations over $B$ with fibres of the homotopy type of $\bar{K} \times \bar{L}$, or Postnikov fibrations over $B$ with distinguished fibre $\bar{K} \times \bar{L}$, or principal $\bar{K} \times \bar{L}$-fibrations over $B$.

(ii) The group action of Proposition 1.5 determines an orbit set

$$
\left(H^{m+1}(B ; G) \oplus H^{n+1}(B ; H)\right) / \mathcal{E}(K(G, m+1) \times K(H, n+1)),
$$

and there is a canonical bijection from this orbit set to $\operatorname{FHT}(K(G, m) \times$ $K(H, n): B)$ that is natural in $B$.

(iii) Let us assume that $\kappa \in H^{m+1}(B ; G)$ corresponds to $[u] \in[B, K(G, m+1)]^{0}$ and $\lambda \in H^{n+1}(B ; H)$ corresponds to $[v] \in[B, K(H, n+1)]^{0}$. Then the bijection of $(i i)$ takes $[(\kappa, \lambda)]$, the orbit of $(\kappa, \lambda)$, to the fibrewise homotopy type of 
Homology, Homotopy and Applications, vol. 8(2), 2006

the principal $\bar{K} \times \bar{L}$-fibration over $B$ induced by the map

$$
B \rightarrow K(G, m+1) \times K(H, n+1), \quad b \mapsto(u(b), v(b))
$$

from the path fibration over $K(G, m+1) \times K(H, n+1)$.

\section{Notations and other Preliminaries.}

We work in the context of the category of compactly generated spaces or $c g$ spaces $[\mathrm{V}]$.

2.1. If $p: X \rightarrow B$ and $f: A \rightarrow B$ are maps, then there is an associated pullback space $A \sqcap(f) X$. The associated projections will be written $p^{*} f: A \sqcap(f) X \rightarrow X$ and $f^{*} p: A \sqcap(f) X \rightarrow A$.

Let $\tau\left(k_{1}, k_{2}\right)$ be a Postnikov tower consisting of the principal fibrations $p_{1}: E_{1} \rightarrow$ $B$ and $p_{2}: E_{2} \rightarrow E_{1}$. Then $E_{1}=B \sqcap\left(k_{1}\right) P K, E_{2}=\left(B \sqcap\left(k_{1}\right) P K\right) \sqcap\left(k_{2}\right) P L$, and $p_{1}$ and $p_{2}$ are the obvious projections.

2.2. Let $M(X, Y)$ and $M^{0}(X, Y)$ denote the spaces of free and based maps from $X$ to $Y$, respectively, in each case carrying the $c g$-ified version of the compact open topology. If $\left(X, X^{\prime}\right)$ and $\left(Y, Y^{\prime}\right)$ are topological pairs, then $M\left(X, X^{\prime} ; Y, Y^{\prime}\right)$ will denote the corresponding mapping space. The path components of $M(X, Y)$ and $M^{0}(X, Y)$ that contain a given map $f$ will be denoted by $M(X, Y ; f)$ and $M^{0}(X, Y ; f)$, respectively.

2.3. Free and based homotopies will be denoted by $\simeq$ and $\simeq^{0}$, respectively, and the set of based homotopy classes of maps from $X$ to $Y$ by $[X, Y]^{0}$.

2.4. The spaces $K(G, m+1), K(H, n+1), K(G, m)$ and $K(H, n)$ will be abbreviated to $K, L, \bar{K}$ and $\bar{L}$, respectively. Thus $\bar{K}=\Omega K$ and $\bar{L}=\Omega L$. Often these spaces require basepoints, in such cases their identities $e$ will play that role.

2.5. The path fibrations over $K$ and $L$ will be denoted by $p: P K \rightarrow K$ and $q: P L \rightarrow$ $L$, respectively.

2.6. If $\theta \in$ Aut $G$, then $\bar{\theta}: \bar{K} \rightarrow \bar{K}$ and $\underline{\theta}: K \rightarrow K$ will be the associated induced homeomorphisms. Then $\underline{\theta}$ induces a homeomorphism $\theta_{\wedge}: P K \rightarrow P K$ by covariant composition. If $\phi \in$ Aut $H$, then $\bar{\phi}: \bar{L} \rightarrow \bar{L}, \underline{\phi}: L \rightarrow L$ and $\phi_{\wedge}: P L \rightarrow P L$ will denote the analagous homeomorphisms for that case.

2.7. We will use the notations $\langle\theta, \psi, \phi\rangle$ and $\langle y, z\rangle$ to denote the matrices

$$
\left(\begin{array}{ll}
\theta & 0 \\
\psi & \phi
\end{array}\right) \quad \text { and } \quad\left(\begin{array}{c}
y \\
z
\end{array}\right)
$$

respectively, where $\theta \in$ Aut $G, \phi \in$ Aut $H, \psi \in M^{0}(\bar{K}, \bar{L}), y \in \bar{K}$, and $z \in \bar{L}$. Matrix multiplication, i.e. the rule

$$
\langle y, z\rangle \mapsto\langle\theta, \psi, \phi\rangle \times\langle y, z\rangle=\langle\theta(y), \psi(y)+\phi(z)\rangle,
$$

determines a based self-homeomorphism of $\bar{K} \times \bar{L}$. 
2.8. We will abbreviate the concept of fibrewise homotopy equivalence (or fibre homotopy equivalence) to FHE. Let $h$ be an FHE between fibrations with distinguished fibres $\bar{K} \times \bar{L}$. Then $h \mid(\bar{K} \times \bar{L})$ is a self-homotopy equivalence of $\bar{K} \times \bar{L}$, and necessarily determines a unique matrix $\langle\theta,[\psi], \phi\rangle$, by restriction to the distinguished fibres (see the discussion before Proposition 1.1). Such an $h$ will be referred to as a $(\theta, \psi, \phi)$ FHE.

In what follows we will make use of a stronger than usual form of FHE, that applies to fibrations with identical distinguished fibres. Two such fibrations, over the same pointed base space, will be said to be 1FHE if there is an FHE between them whose restriction to those fibres is homotopic to the identity on that space. Equivalently, if the base space is well pointed, we can require that there exists an FHE whose restriction to the distinguished fibres is the identity on that space (see $[\mathrm{B} 1,(2.4 .6)])$. We will denote the set of 1-fibrewise homotopy types of fibrations over $B$ with distinguished fibres $F$ by $1 \mathrm{FHT}(F: B)$.

We notice that a $1 \mathrm{FHE}$, between fibrations with distinguished fibres $\bar{K} \times \bar{L}$, is an example of a $(1,0,1)$ FHE.

The following standard results will be quoted repeatedly in what follows.

2.9. Let $A_{0} \subset A$ be a cofibration, and $K, L: A \times I \rightarrow W$ be homotopies that extend a given map $N:(A \times\{0\}) \cup\left(A_{0} \times I\right) \rightarrow W$. Then $K|A \times\{1\} \simeq L| A \times\{1\}$ relative to $A_{0} \times\{1\}$, i.e. this last homotopy extends the homotopy

$$
A_{0} \times I \rightarrow W, \quad(a, t) \mapsto N(a, 1),
$$

where $a \in A_{0}$ and $t \in I$ (this is immediate from [W, Ch.1, Thm.7.18], with $B$ of that result taken to be a point).

2.10. Let $r: Z \rightarrow B$ be a fibration and $H: A \times I \rightarrow B$ be a homotopy from $f$ to $g$. It is well known that there is an FHE $h: A \sqcap(f) Z \rightarrow A \sqcap(g) Z$ over $A$ [S, Thm.2.8.14].

\section{Group Actions on Sets of Postnikov Fibrations}

In this section, we introduce group actions of Aut $H$, Aut $G$ and $[\bar{K}, \bar{L}]^{0}$ on $1 \mathrm{FHT}(\bar{K} \times \bar{L}, B)$, and blend them together to produce the action of $\mathcal{E}(\bar{K} \times \bar{L})$ referred to in Proposition 1.1. The proofs of the Lemmas used are removed to Section 9.

Let $\phi \in$ Aut $H$ and the Postnikov fibration $t\left(k_{1}, k_{2}\right)$ be over $B$ and have distinguished fibre $\bar{K} \times \bar{L}$. Then we can define an associated Postnikov fibration over $B$, as follows:

$$
\phi \cdot t\left(k_{1}, k_{2}\right)=t\left(k_{1}, \underline{\phi} \circ k_{2}\right)
$$

If follows via [B1, 2.7.7] that $\phi \cdot t\left(k_{1}, k_{2}\right)$ also has distinguished fibre $\bar{K} \times \bar{L}$.

Lemma 3.2. (i) There is a group action

$$
\text { Aut } H \times 1 \mathrm{FHT}(\bar{K} \times \bar{L}: B) \rightarrow 1 \mathrm{FHT}(\bar{K} \times \bar{L}: B),
$$

$\left(\phi,\left[t\left(k_{1}, k_{2}\right)\right]\right) \mapsto\left[\phi \cdot t\left(k_{1}, k_{2}\right)\right]$, where $\phi \in$ Aut $H$ and the Postnikov fibration $t\left(k_{1}, k_{2}\right)$ is over $B$ and has distinguished fibre $\bar{K} \times \bar{L}$. 
(ii) There is a $(1,0, \phi) \mathrm{FHE}$ from $t\left(k_{1}, k_{2}\right)$ to $\phi \cdot t\left(k_{1}, k_{2}\right)$, i.e. the homeomorphism

$$
\Phi=\Phi(\phi):\left(B \sqcap\left(k_{1}\right) P K\right) \sqcap\left(k_{2}\right) P L \rightarrow\left(B \sqcap\left(k_{1}\right) P K\right) \sqcap\left(\underline{\phi} \circ k_{2}\right) P L,
$$

with $\Phi(b, y, z)=\left(b, y, \phi_{\wedge}(z)\right)$, for $(b, y, z) \in\left(B \sqcap\left(k_{1}\right) P K\right) \sqcap\left(k_{2}\right) P L$.

Let $\theta \in \operatorname{Aut}(G)$ and $t\left(k_{1}, k_{2}\right)$ be a Postnikov fibration over $B$ with distinguished fibre $\bar{K} \times \bar{L}$. We notice that there is a homeomorphism

$$
1 \sqcap \theta_{\wedge}: B \sqcap\left(k_{1}\right) P K \rightarrow B \sqcap\left(\underline{\theta} \circ k_{1}\right) P K,
$$

with inverse $1 \sqcap \theta_{\wedge}^{-1}$. Then we can define an associated Postnikov fibration, again over $B$ with distinguished fibre $\bar{K} \times \bar{L}$, i.e.

$$
\theta \cdot t\left(k_{1}, k_{2}\right)=t\left(\underline{\theta} \circ k_{1}, k_{2} \circ\left(1 \sqcap \theta_{\wedge}^{-1}\right)\right)
$$

Lemma 3.4. (i) There is a group action

$$
\text { Aut } G \times 1 \mathrm{FHT}(\bar{K} \times \bar{L}: B) \rightarrow 1 \mathrm{FHT}(\bar{K} \times \bar{L}: B),
$$

$\left(\theta,\left[t\left(k_{1}, k_{2}\right)\right]\right) \mapsto\left[\theta \cdot t\left(k_{1}, k_{2}\right)\right]$, where $\theta \in$ Aut $G$ and the Postnikov fibration $t\left(k_{1}, k_{2}\right)$ is over $B$ and has distinguished fibre $\bar{K} \times \bar{L}$.

(ii) There is a $(\theta, 0,1) \mathrm{FHE}$ from $t\left(k_{1}, k_{2}\right)$ to $\theta \cdot t\left(k_{1}, k_{2}\right)$, i.e. the homeomorphism

$$
\begin{aligned}
& \Theta=\Theta(\theta):\left(B \sqcap\left(k_{1}\right) P K\right) \sqcap\left(k_{2}\right) P L \rightarrow\left(B \sqcap\left(\underline{\theta} \circ k_{1}\right) P K\right) \sqcap\left(k_{2} \circ\left(1 \sqcap \theta_{\wedge}^{-1}\right)\right) P L, \\
& (b, y, z) \mapsto\left(b, \theta_{\wedge}(y), z\right), \text { for }(b, y, z) \in\left(B \sqcap\left(k_{1}\right) P K\right) \sqcap\left(k_{2}\right) P L .
\end{aligned}
$$

We will now consider the action of a third group whose elements appear in several different guises. It is standard, of course, that $H^{n}(\bar{K} ; H)$ can be identified with $[\bar{K}, \bar{L}]^{0}$. Viewing $\bar{L}$ as $\Omega L$, we can interpret the members of the above group - or, to be more precise, the maps that make up the aforementioned members in several different ways.

Lemma 3.5. (i) There are bijections between the sets of:

(a) based maps $\psi: \bar{K} \rightarrow \bar{L}$,

(b) maps $\psi_{1}:(\bar{K} \times I,(\bar{K} \times\{0,1\}) \cup(\{e\} \times I)) \rightarrow(L,\{e\})$, and

(c) maps $\psi_{2}: I \rightarrow M^{0}(\bar{K}, L ; c(e))$ such that $\psi_{2}(0)=\psi_{2}(1)=c(e)$.

determined by the rule $\psi(z)(t)=\psi_{1}(z, t)=\psi_{2}(t)(z)$, where $z \in \bar{K}$ and $t \in I$.

(ii) The above carries over to homotopy classes. Thus there are bijective correspondences $[\psi] \leftrightarrow\left[\psi_{1}\right] \leftrightarrow\left[\psi_{2}\right]$, where $[-]$ denotes homotopy class, in each case defined in the sense appropriate to the maps in question.

Let $\psi: \bar{K} \rightarrow \bar{L}$ be a based map, and $t\left(k_{1}, k_{2}\right)$ a Postnikov fibration over $B$ with distinguished fibre $\bar{K} \times \bar{L}$. It follows, via the cofibration property for $\left\{b_{0}\right\} \times \bar{K} \subset$ $B \sqcap\left(k_{1}\right) P K$ (see [B1, 2.4.4 and 2.4.8]) that there is a homotopy

$$
S=S\left(k_{1}, k_{2}, \psi\right):\left(B \sqcap\left(k_{1}\right) P K\right) \times I \rightarrow L
$$

that extends the second $k$-invariant $k_{2}:\left(B \sqcap\left(k_{1}\right) P K\right) \times\{0\}=\left(B \sqcap\left(k_{1}\right) P K\right) \rightarrow L$ as well as the homotopy $\psi_{1}:\left\{b_{0}\right\} \times \bar{K} \times I \cong \bar{K} \times I \rightarrow L$. Let $\psi \cdot k_{2}: E_{1} \rightarrow L$ be determined by the rule $\left(\psi \cdot k_{2}\right)(b, y)=S(b, y, 1)$, where $k_{1}(b)=p(y)$. We define an associated Postnikov fibration, i.e. 


$$
\psi \cdot t\left(k_{1}, k_{2}\right)=t\left(k_{1}, \psi \cdot k_{2}\right),
$$

which again is over $B$ and has distinguished fibre $\bar{K} \times \bar{L}$. Then $\left(\psi \cdot k_{2}\right)\left(b_{0}, y\right)=$ $S\left(b_{0}, y, 1\right)=\psi_{1}\left(b_{0}, y, 1\right)=e$. So $\left(\psi \cdot k_{2}\right)\left(\left\{b_{0}\right\} \times \bar{K}\right)=e$ and it follows [B1, (2.7.7)] that $\psi \cdot t\left(k_{1}, k_{2}\right)$ also has distinguished fibre $\bar{K} \times \bar{L}$.

The map $\psi \cdot k_{2}$ is not uniquely determined, but the homotopy class $[\psi] \cdot\left[k_{2}\right]=$ $\left[\psi \cdot k_{2}\right]$ in $\left[\left(B \sqcap\left(k_{1}\right) P K\right),\left\{b_{0}\right\} \times \bar{K} ; L, e\right]$ is so defined (see $\left.(2.9)\right)$. It follows, via the relative version of $(2.10)$, that $t\left(k_{1}, \psi \cdot k_{2}\right)$ is unique up to $1 \mathrm{FHT}$.

Lemma 3.8. (i) There is a group action

$$
[\bar{K}, \bar{L}]^{0} \times 1 \mathrm{FHT}(\bar{K} \times \bar{L}: B) \rightarrow 1 \mathrm{FHT}(\bar{K} \times \bar{L}: B),
$$

defined by $\left([\psi],\left[t\left(k_{1}, k_{2}\right)\right]\right) \mapsto\left[\psi \cdot t\left(k_{1}, k_{2}\right)\right]$.

(ii) Let $t\left(k_{1}, k_{2}\right)$ be a Postnikov fibration over $B$ with distinguished fibre $\bar{K} \times \bar{L}$, and $\psi \in M^{0}(\bar{K}, \bar{L})$. Then there is a $(1, \psi, 1) \mathrm{FHE}$ from $t\left(k_{1}, k_{2}\right)$ to $\psi \cdot t\left(k_{1}, k_{2}\right)$, i.e.

$$
\Psi=\Psi(\psi):\left(B \sqcap\left(k_{1}\right) P K\right) \sqcap\left(k_{2}\right) P L \rightarrow\left(B \sqcap\left(k_{1}\right) P K\right) \sqcap\left(\psi \cdot k_{2}\right) P L .
$$

We are almost ready to blend together the actions of Aut $G$, Aut $H$ and $[\bar{K}, \bar{L}]^{0}$ on $1 \mathrm{FHT}(\bar{K} \times \bar{L}: B)$. We must first, however, determine the extent to which these actions commute with each other.

Lemma 3.9. Let $\theta, \theta^{\prime} \in$ Aut $G, \phi, \phi^{\prime} \in$ Aut $H, \psi, \psi^{\prime} \in M^{0}(\bar{K}, \bar{L})$ and $t\left(k_{1}, k_{2}\right)$ be a Postnikov fibration over $B$ that has distinguished fibre $\bar{K} \times \bar{L}$. Then:

(i) $\phi^{\prime} \cdot\left(\phi \cdot t\left(k_{1}, k_{2}\right)\right)$ is 1FHE to $\left(\phi^{\prime} \circ \phi\right) \cdot t\left(k_{1}, k_{2}\right)$,

(ii) $\theta^{\prime} \cdot\left(\theta \cdot t\left(k_{1}, k_{2}\right)\right)$ is $1 \mathrm{FHE}$ to $\left(\theta^{\prime} \circ \theta\right) \cdot t\left(k_{1}, k_{2}\right)$,

(iii) $\psi^{\prime} \cdot\left(\psi \cdot t\left(k_{1}, k_{2}\right)\right)$ is $1 \mathrm{FHE}$ to $\left(\psi^{\prime}+\psi\right) \cdot t\left(k_{1}, k_{2}\right)$,

(iv) $\theta \cdot\left(\phi \cdot t\left(k_{1}, k_{2}\right)\right)$ is $1 \mathrm{FHE}$ to $\phi \cdot\left(\theta \cdot t\left(k_{1}, k_{2}\right)\right)$,

(v) $\psi \cdot\left(\theta \cdot t\left(k_{1}, k_{2}\right)\right)$ is $1 \mathrm{FHE}$ to $\theta \cdot\left((\psi \circ \bar{\theta}) \cdot t\left(k_{1}, k_{2}\right)\right)$, and

(vi) $\phi \cdot\left(\psi \cdot t\left(k_{1}, k_{2}\right)\right)$ is $1 \mathrm{FHE}$ to $(\bar{\phi} \circ \psi) \cdot\left(\phi \cdot t\left(k_{1}, k_{2}\right)\right)$.

Let $\theta \in$ Aut $G, \psi \in M^{0}(\bar{K}, \bar{L}), \phi \in$ Aut $H$, and $t\left(k_{1}, k_{2}\right)$ be a Postnikov fibration over $B$ with distinguished fibre $\bar{K} \times \bar{L}$. We define

$$
\langle\theta, \psi, \phi\rangle \cdot t\left(k_{1}, k_{2}\right)=\theta \cdot\left(\psi \cdot\left(\phi \cdot t\left(k_{1}, k_{2}\right)\right)\right) .
$$

The actions of $\operatorname{Aut}(G), \operatorname{Aut}(H)$, and $[\bar{K}, \bar{L}]^{0}$ all preserve both the base space $B$ and the property of having distinguished fibre $\bar{K} \times \bar{L}$, hence the Postnikov fibration $\langle\theta, \psi, \phi\rangle \cdot t\left(k_{1}, k_{2}\right)$ also has these same properties.

Proposition 3.11. (i) There is a group action

$$
\begin{aligned}
\mathcal{E}(\bar{K} \times \bar{L}) \times 1 \mathrm{FHT}(\bar{K} \times \bar{L}: B) & \rightarrow 1 \mathrm{FHT}(\bar{K} \times \bar{L}: B) \\
\left(\langle\theta,[\psi], \phi\rangle,\left[t\left(k_{1}, k_{2}\right)\right]\right) & \mapsto\left[\langle\theta, \psi, \phi\rangle \cdot t\left(k_{1}, k_{2}\right)\right],
\end{aligned}
$$

where $\langle\theta,[\psi], \phi\rangle \in \mathcal{E}(\bar{K} \times \bar{L})$ and $t\left(k_{1}, k_{2}\right)$ is a Postnikov fibration over $B$ with distinguished fibre $\bar{K} \times \bar{L}$. 
(ii) If $t\left(k_{1}, k_{2}\right)$ is a Postnikov fibration over $B$ with distinguished fibre $\bar{K} \times \bar{L}$, then there is a $(\theta, \psi, \phi) \mathrm{FHE}$ from $t\left(k_{1}, k_{2}\right)$ to $\langle\theta, \psi, \phi\rangle \cdot t\left(k_{1}, k_{2}\right)$.

(iii) Let $t\left(k_{1}, k_{2}\right)$ and $t\left(\ell_{1}, \ell_{2}\right)$ be Postnikov fibrations over $B$, each with distinguished fibre $\bar{K} \times \bar{L}$. Then $t\left(k_{1}, k_{2}\right)$ is FHE to $t\left(\ell_{1}, \ell_{2}\right)$ if and only if there exists $\theta \in$ Aut $G, \psi \in M^{0}(\bar{K}, \bar{L})$, and $\phi \in$ Aut $H$ such that $\langle\theta, \psi, \phi\rangle \cdot t\left(k_{1}, k_{2}\right)$ is $1 \mathrm{FHE}$ to $t\left(\ell_{1}, \ell_{2}\right)$.

Proof. (i) Let $\theta, \theta^{\prime} \in$ Aut $G, \phi, \phi^{\prime} \in$ Aut $H$ and $\psi, \psi^{\prime} \in M^{0}(\bar{K}, \bar{L})$, with $\langle\theta, \psi, \phi\rangle$ and $\left\langle\theta^{\prime}, \psi^{\prime}, \phi^{\prime}\right\rangle$ denoting the associated $2 \times 2$ matrices, and $t\left(k_{1}, k_{2}\right)$ be a Postnikov fibration of the required type. Then it follows from Lemma 3.9 that the following fibrations are $1 \mathrm{FHE}$ :

(a) $\left\langle\theta^{\prime}, \psi^{\prime}, \phi^{\prime}\right\rangle \cdot\left(\langle\theta, \psi, \phi\rangle \cdot t\left(k_{1}, k_{2}\right)\right)$,

(b) $\theta^{\prime} \cdot \psi^{\prime} \cdot \phi^{\prime} \cdot \theta \cdot \psi \cdot \phi \cdot t\left(k_{1}, k_{2}\right)$ by 3.10

(c) $\theta^{\prime} \cdot \psi^{\prime} \cdot \theta \cdot \phi^{\prime} \cdot \psi \cdot \phi \cdot t\left(k_{1}, k_{2}\right)$ by (iv) of 3.9 ,

(d) $\theta^{\prime} \cdot \theta \cdot\left(\psi^{\prime} \circ \bar{\theta}\right) \cdot\left(\overline{\phi^{\prime}} \circ \psi\right) \cdot \phi^{\prime} \cdot \phi \cdot t\left(k_{2}, k_{2}\right)$ by $3.9(\mathrm{v})$ and $(\mathrm{vi})$,

(e) $\left(\theta^{\prime} \circ \theta\right) \cdot\left(\psi^{\prime} \circ \bar{\theta}+\bar{\phi}^{\prime} \circ \psi\right) \cdot\left(\phi^{\prime} \circ \phi\right) \cdot t\left(k_{1}, k_{2}\right)$ by $3.9(\mathrm{i})$, (ii) and (iii), and

(f) $\left\langle\theta^{\prime} \circ \theta, \psi^{\prime} \circ \bar{\theta}+\overline{\phi^{\prime}} \circ \psi, \phi^{\prime} \circ \phi\right\rangle \cdot t\left(k_{1}, k_{2}\right)$ by 3.10 .

Now $\left\langle\theta^{\prime}, \psi^{\prime}, \phi^{\prime}\right\rangle \times\langle\theta, \psi, \phi\rangle=\left\langle\theta^{\prime} \circ \theta, \psi^{\prime} \circ \bar{\theta}+\overline{\phi^{\prime}} \circ \psi, \phi^{\prime} \cdot \phi\right\rangle$, so we do have an action.

(ii) There are $(\theta, 0,1),(1, \psi, 1)$ and $(1,0, \phi) \mathrm{FHE} s$, i.e. $\Theta$ from $\psi \cdot \phi \cdot t\left(k_{1}, k_{2}\right)$ to $\theta \cdot \psi \cdot \phi \cdot t\left(k_{1}, k_{2}\right), \Psi$ from $\phi \cdot t\left(k_{1}, k_{2}\right)$ to $\psi \cdot \phi \cdot t\left(k_{1}, k_{2}\right)$, and $\Phi$ from $t\left(k_{1}, k_{2}\right)$ to $\phi \cdot t\left(k_{1}, k_{2}\right)$ (see parts (ii) of Lemmas 3.4, 3.8 and 3.2, respectively). Multiplying matrices

$$
\langle\theta, 0,1\rangle \times\langle 1, \psi, 1\rangle \times\langle 1,0, \phi\rangle=\langle\theta, \psi, \phi\rangle,
$$

so $\Theta \circ \Psi \circ \Phi: t\left(k_{1}, k_{2}\right) \rightarrow\langle\theta, \psi, \phi\rangle \cdot t\left(k_{1}, k_{2}\right)$ is a $(\theta, \psi, \phi) \mathrm{FHE}$.

(iii) The "if" condition is immediate from the data and (ii). Now an FHE from $t\left(k_{1}, k_{2}\right)$ to $t\left(\ell_{1}, \ell_{2}\right)$ is a $(\theta, \psi, \phi) \mathrm{FHE}$, for suitable choices of $\theta \in$ Aut $G, \psi \in M^{0}(\bar{K}, \bar{L})$ and $\phi \in$ Aut $H$ (see (2.8)). The result follows after applying (ii) to $t\left(k_{1}, k_{2}\right)$.

Theorem 3.12 (Preliminary Classification Theorem). Let $m$ and $n$ be integers such that $0<m<n, G$ and $H$ be Abelian groups, and $B$ be a well pointed space. Then there is a bijection:

$$
1 \mathrm{FHT}(\bar{K} \times \bar{L}: B) / \mathcal{E}(\bar{K} \times \bar{L}) \rightarrow \mathrm{FHT}(\bar{K} \times \bar{L}: B)
$$

determined by the rule $\left[\left[t\left(k_{1}, k_{2}\right)\right]\right] \mapsto\left[t\left(k_{1}, k_{2}\right)\right]$. Thus if $t\left(k_{1}, k_{2}\right)$ is a Postnikov fibration over $B$ with distinguished fibre $\bar{K} \times \bar{L}$, the orbit $\left[\left[t\left(k_{1}, k_{2}\right)\right]\right]$ of the $1 \mathrm{FHT}$ class of $t\left(k_{1}, k_{2}\right)$ corresponds to the FHT class $\left[t\left(k_{1}, k_{2}\right)\right]$ of $t\left(k_{1}, k_{2}\right)$. The bijection is natural relative to $B$.

Proof. This is immediate from (iii) of Proposition 3.11.

\section{Classifying Spaces and Exponential Laws}

The next part of our main argument requires the use of the fibred or freerange mapping space $M_{\infty}$, and a fibred or freerange exponential law. We give a few basic 
ideas concerning these concepts here; the reader can find more information in [B1] and [B3].

\subsection{Classifying Spaces.}

We define the set $M_{\infty}$ as the set of null homotopic maps from individual fibres of $p$ to $L$, i.e. $\bigcup_{x \in K} M(P K \mid x, L ; c(e))$. The function $p_{\infty}: M_{\infty} \rightarrow K$ is the projection that takes maps $P K \mid x \rightarrow L$ to $x$, where $x \in K$.

We topologize $M_{\infty}$ with the $c g$-ification of the topology that has subbasis consisting of all sets of maps of the forms:

(i) $p_{\infty}^{-1}(U)$, where $U$ is open in $K$, and

(ii) $W(C, V)=\bigcup_{x \in K}\{f \in M(P K \mid x, L ; c(e)) \mid f(C \cap(P K \mid x)) \subset V\}$,

where $C$ is compact in $P K$ and $V$ is open in $L$. Then $p_{\infty}$ is obviously continuous, in fact it is a fibration (see (M3) of [B1, p.90]).

The distinguished fibre of $p_{\infty}$ is $M(\bar{K}, L ; c(e))$ (see (M4) of [B1, p.90]). We will use $i_{\infty}: M(\bar{K}, L ; c(e)) \rightarrow M_{\infty}$ to denote the corresponding inclusion map. The constant map $c(e): \bar{K} \rightarrow L$ will be taken as basepoint for both $M(\bar{K}, L ; c(e))$ and $M_{\infty}$.

\subsection{A Fibred Exponential Law and Classifying Maps.}

Let $B$ be a pointed weak Hausdorff space (see [B1, p.91]), and $k_{1}: B \rightarrow K$ be a fixed pointed map. The following rule is (M6) of [B1, p.90].

There is a bijective correspondence between:

(i) the set of pointed maps $k: B \rightarrow M_{\infty}$ such that $p_{\infty} \circ k=k_{1}$, and

(ii) the set of maps $k_{2}: B \sqcap\left(k_{1}\right) P K \rightarrow L$, extending $c(e):\left\{b_{0}\right\} \times \bar{K} \rightarrow L$, determined by the rule

$$
k(b)(y)=k_{2}(b, y) \text {, where } k_{1}(b)=p(y) .
$$

If we allow $k_{1}$ to vary through $M^{0}\left(B, M_{\infty}\right)$, we obtain a bijective correspondence between

(i) the set of based maps $k: B \rightarrow M_{\infty}$, and

(ii) the set of Postnikov fibrations over $B$ with distinguished fibres $\bar{K} \times \bar{L}$

determined by the rule $k \mapsto t\left(p_{\infty} \circ k, k_{2}\right)$, where $k_{2}$ is determined by $k$ as specified previously.

In that situation the map $k$ will be referred to as the classifying map for $t\left(k_{1}, k_{2}\right)$.

\subsection{Universal Postnikov Fibrations.}

The maps $p_{\infty}: M_{\infty} \rightarrow K$ and $p: P K \rightarrow K$ determine a pullback space $M_{\infty} \sqcap$ $\left(p_{\infty}\right) P K$, and there is an associated evaluation map

$$
e_{\infty}: M_{\infty} \sqcap\left(p_{\infty}\right) P K \rightarrow L, \quad(f, y) \mapsto f(y),
$$

where $p_{\infty}(f)=p(y)$. We recall, from (M9) of [B1, p.90], that the Postnikov fibration $t\left(p_{\infty}, e_{\infty}\right)$ is universal amongst Postnikov fibrations. Thus, for each $t\left(k_{1}, k_{2}\right)$ over $B$ 
with distinguished fibre $\bar{K} \times \bar{L}, t\left(k_{1}, k_{2}\right)=k^{*} t\left(p_{\infty}, e_{\infty}\right)$, and so the rule $k \mapsto t\left(k_{1}, k_{2}\right)$ can alternatively be defined as $k \mapsto k^{*} t\left(p_{\infty}, e_{\infty}\right)$.

\subsection{A 1FHT Classification Theorem.}

Let $m$ and $n$ be integers with $1<m<n, G$ and $H$ be Abelian groups, and $B$ be a well pointed space having the homotopy type of a simply connected CW-complex. Then [B1, Theorem 7.5] asserts that there is a bijection

$$
\left[B, M_{\infty}\right]^{0} \rightarrow 1 \mathrm{FHT}(\bar{K} \times \bar{L}: B),
$$

determined by the rule $[k] \rightarrow\left[t\left(k_{1}, k_{2}\right)\right]$ of (4.2).

The Postnikov fibration $t\left(p_{\infty}, e_{\infty}\right)$ is universal amongst Postnikov fibrations over $B$ with distinguished fibre $\bar{K} \times \bar{L}$, in the sense that the rule $[k] \mapsto\left[k^{*} t\left(p_{\infty}, e_{\infty}\right)\right]$ defines the last bijection.

\section{The Basic FHT Classification Theorem}

In this section, we use (4.4) to replace the orbit set $1 \mathrm{FHT}(\bar{K} \times \bar{L}: B) / \mathcal{E}(\bar{K} \times \bar{L})$ of Theorem 3.12 by a more computable orbit set $\left[B, M_{\infty}\right]^{0} / \mathcal{E}(\bar{K} \times \bar{L})$.

If $k \in M^{0}\left(B, M_{\infty}\right)$, we recall that $k(b): P K \mid k_{1}(b) \rightarrow L$ and then define $\phi \cdot k \in$ $M^{0}\left(B, M_{\infty}\right)$ by

5.1 .

$$
(\phi \cdot k)(b)=\underline{\phi} \circ k(b): P K \mid k_{1}(b) \rightarrow L \text { where } b \in B .
$$

Lemma 5.2. (i) There is a group action

$$
\text { Aut } H \times\left[B, M_{\infty}\right]^{0} \rightarrow\left[B, M_{\infty}\right]^{0}, \quad(\phi,[k]) \mapsto[\phi \cdot k],
$$

where $\phi \in$ Aut $H$ and $k \in M^{0}\left(B, M_{\infty}\right)$.

(ii) Let $t\left(k_{1}, k_{2}\right)$ be a Postnikov fibration over $B$ with distinguished fibre $\bar{K} \times \bar{L}$ and $\phi \in \operatorname{Aut}(H)$. Then $t\left(k_{1}, k_{2}\right)$ is classified by $k$ if and only if $\phi \cdot t\left(k_{1}, k_{2}\right)$ is classified by $\phi \cdot k$.

Proof. (i) $\left(\phi_{1} \cdot\left(\phi_{2} \cdot k\right)\right)(b)=\phi_{1} \circ\left(\phi_{2} \circ k(b)\right)=\left(\phi_{1} \circ \phi_{2}\right) \circ k(b)=\left(\phi_{1} \circ \phi_{2}\right) \circ k(b)=$ $\left(\left(\phi_{1} \circ \phi_{2}\right) \cdot k\right)(b)$. Hence $\phi_{1} \cdot\left(\overline{\phi_{2}} \cdot k\right)=\left(\phi_{1} \circ \phi_{2}\right) \cdot k$.

(ii) If $t\left(k_{1}, k_{2}\right)$ is classified by $k$, then the Postnikov fibration $\phi \cdot t\left(k_{1}, k_{2}\right)$ has second $k$-invariant $\underline{\phi} \circ k_{2}$, and $\left(\phi \circ k_{2}\right)(b, y)=\phi(k(b)(y))=(\phi \cdot k)(b)(y)$, where $(b, y) \in$ $B \sqcap\left(k_{1}\right) P K$. Further, $\left.p_{\infty} \circ \overline{(\phi} \cdot k\right)=k_{1}$, and so $\phi \cdot t\left(k_{1}, k_{2}\right)$ is classified by $\phi \cdot k$. The argument is reversible, so the proof is complete.

If $\theta \in$ Aut $G$, then there is a based homeomorphism $\theta_{\wedge}^{-1}: P K \rightarrow P K$ over $\underline{\theta}^{-1}: K \rightarrow$ $K$ (2.6). So, if $x \in K$, we then have a homeomorphism $\theta_{\wedge}^{-1}|(P K \mid \underline{\theta}(x)): P K| \underline{\theta}(x) \rightarrow$ $P K \mid x$. If $k \in M^{0}\left(B, M_{\infty}\right)$, then $k_{1}=p_{\infty} \circ k \in M^{0}(B, K)$, and we can define $\theta \cdot k \in$ $M^{0}\left(B, M_{\infty}\right)$ by

$$
(\theta \cdot k)(b)=k(b) \circ\left(\theta_{k_{1}(b)}^{-1} \mid\left(P K \mid\left(\underline{\theta} \circ k_{1}\right)(b)\right)\right) .
$$


Lemma 5.4. (i) There is a group action

$$
\text { Aut } G \times\left[B, M_{\infty}\right]^{0} \rightarrow\left[B, M_{\infty}\right]^{0}, \quad(\theta,[k]) \mapsto[\theta \cdot k],
$$

where $\theta \in$ Aut $G$ and $k \in M^{0}\left(B, M_{\infty}\right)$.

(ii) Let $t\left(k_{1}, k_{2}\right)$ be a Postnikov fibration over $B$ with distinguished fibre $\bar{K} \times \bar{L}$ and $\theta \in \operatorname{Aut}(G)$. Then $t\left(k_{1}, k_{2}\right)$ is classified by $k$ if and only if $\theta \cdot t\left(k_{1}, k_{2}\right)$ is classified by $\theta \cdot k$.

Proof. (i) $\left(\theta_{1} \cdot\left(\theta_{2} \cdot k\right)\right)(b)$

$$
\begin{aligned}
& =\left(\theta_{2} \cdot k\right)(b) \circ\left(\theta_{1}\right)_{\wedge}^{-1} \mid\left(P K \mid\left(\underline{\theta}_{1} \circ \underline{\theta}_{2} \circ k_{1}\right)(b)\right) \\
& =\left(k(b) \circ\left(\theta_{2}\right)_{\wedge}^{-1} \mid\left(P K \mid\left(\underline{\theta}_{2} \circ k_{1}\right)(b)\right)\right) \circ\left(\left(\theta_{1}\right)_{\wedge}^{-1}|P K|\left(\left(\underline{\theta}_{1} \circ \underline{\theta}_{2} \circ k_{1}\right)(b)\right)\right) \\
& =k(b) \circ\left(\theta_{1} \circ \theta_{2}\right)_{\wedge}^{-1} \mid\left(P K \mid\left(\left(\underline{\theta}_{1} \circ \underline{\theta}_{2} \circ k_{1}\right)(b)\right)\right) \\
& =\left(\left(\theta_{1} \cdot \theta_{2}\right) \cdot k\right)(b) .
\end{aligned}
$$

Hence $\theta_{1} \cdot\left(\theta_{2} \cdot k\right)=\left(\theta_{1} \circ \theta_{2}\right) \cdot k$.

(ii) Let us assume that $k$ classifies $t\left(k_{1}, k_{2}\right)$. Then $(\theta \cdot k)(b)(y)=k(b) \theta_{\wedge}^{-1}(y)=$ $k_{2}\left(b, \theta_{\wedge}^{-1}(y)\right)=k_{2}\left(1 \sqcap \theta_{\wedge}^{-1}\right)(b, y)$. Also $(\theta \cdot k)(b): P K \mid \underline{\theta} k_{1}(b) \rightarrow L$, so $p_{\infty} \circ(\theta \cdot k)=$ $\underline{\theta} \circ k_{1}$. Hence $\theta \cdot k$ classifies $t\left(\underline{\theta} \circ k_{1}, k_{2} \circ\left(1 \sqcap \theta_{\wedge}^{-1}\right)\right)=\theta \cdot t\left(k_{1}, k_{2}\right)$.

Let $\psi \in M^{0}(\bar{K}, \bar{L})$ and $k \in M^{0}\left(B, M_{\infty}\right)$. Identifying $B$ with $B \times\{0\}$ and $I$ with $\left\{b_{0}\right\} \times I$, we can consider $\psi_{2}$ to be a map $\left\{b_{0}\right\} \times I \rightarrow M^{0}(\bar{K}, L ; c(e))$ and $k \in M^{0}\left(B \times\{0\}, M_{\infty}\right)$. Now both $k$ and $\psi_{2}$ take the value $c(e)$ at $\left(b_{0}, 0\right)$, so the non-degeneracy of $\left\{b_{0}\right\} \subset B$ [B1, (2.4.8)] allows us to apply the homotopy extension property. We obtain a homotopy

$$
T=T(k, \psi): B \times I \rightarrow M_{\infty}
$$

that extends both $k$ and $i_{\infty} \circ \psi_{2}$ (see (4.1) for $i_{\infty}$ ). Restricting $T$ to $B=B \times\{1\}$, we define a based map

5.6.

$$
\psi \cdot k: B \rightarrow M_{\infty}, \quad(\psi \cdot k)(b)=T(b, 1), \text { where } b \in B .
$$

As with $\psi \cdot k_{2}$, the map $\psi \cdot k$ is not uniquely determined, but it is unique up to based homotopy.

Lemma 5.7. (i) There is a group action

$$
[\bar{K}, \bar{L}]^{0} \times\left[B, M_{\infty}\right]^{0} \rightarrow\left[B, M_{\infty}\right]^{0}, \quad([\psi],[k]) \mapsto[\psi \cdot k] .
$$

(ii) Let $t\left(k_{1}, k_{2}\right)$ be a Postnikov fibration over $B$ with distinguished fibre $\bar{K} \times \bar{L}$, and $\psi \in M^{0}\left((\bar{K}, \bar{L})\right.$. Then $t\left(k_{1}, k_{2}\right)$ is classified by $k$ if and only if $\psi \cdot t\left(k_{1}, k_{2}\right)$ is classified by $\psi \cdot k$.

Proof. (i) This is similar to the proof of Proposition 3.8(i), which has been removed to Section 9.

(ii) The composition of $k_{1}$ and the projection $\pi: B \times I \rightarrow B$ determines a homotopy $k_{1} \circ \pi: B \times I \rightarrow K$. Pulling $p: P K \rightarrow K$ back over this homotopy defines $(B \times$ $I) \sqcap\left(k_{1} \circ \pi\right) P K$. Identifying this pullback space with $\left(B \sqcap\left(k_{1}\right) P K\right) \times I$, and applying the fibred exponential law to $S$ of (3.6), we obtain a homotopy $S^{<}: B \times I \rightarrow M_{\infty}$ 
that agrees with $T$ on $B \times\{0\}$, i.e. both start at $k: B \times\{0\}=B \rightarrow M_{\infty}$. It follows via (2.9) that the classifying map for $t\left(k_{1}, \psi \cdot k_{2}\right)$, i.e. $S^{<} \mid B \times\{1\}$, is based homotopic to $\psi \cdot k$. The above argument is reversible, so the proof is complete.

We are now ready to specify the action of $\mathcal{E}(\bar{K} \times \bar{L})$ on $\left[B, M_{\infty}\right]^{0}$ that determines the orbit set used in our basic classification theorem.

If $k \in M^{0}\left(B, B_{\infty}\right)$, then we define

$$
\langle\theta, \psi, \phi\rangle \cdot k=\theta \cdot(\psi \cdot(\phi \cdot k)) \in M^{0}\left(B, M_{\infty}\right) .
$$

It follows from (ii) of Lemmas 5.2, 5.4 and 5.7 , that $\langle\theta, \psi, \phi\rangle \cdot k$ is unique up to based homotopy.

Proposition 5.9. (i) There is a group action

$$
\mathcal{E}(\bar{K} \times \bar{L}) \times\left[B, M_{\infty}\right]^{0} \rightarrow\left[B, M_{\infty}\right]^{0},(\langle\theta,[\psi], \phi\rangle,[k]) \mapsto[\langle\theta, \psi, \phi\rangle \cdot k]
$$

(ii) Let $t\left(k_{1}, k_{2}\right)$ be a Postnikov fibration over $B$ with distinguished fibre $\bar{K} \times \bar{L}$. The actions of Propositions 3.11(i) and 5.9(i) agree in the sense that $t\left(k_{1}, k_{2}\right)$ is classified by $k$ if, and only if, $\langle\theta, \psi, \phi\rangle \cdot t\left(k_{1}, k_{2}\right)$ is classified by $\langle\theta, \psi, \phi\rangle \cdot k$.

Proof. We will prove (ii) first. Let us consider:

(a) $t\left(k_{1}, k_{2}\right)$ is classified by $k$,

(b) $\phi \cdot t\left(k_{1}, k_{2}\right)$ is classified by $\phi \cdot k$,

(c) $\psi \cdot \phi \cdot t\left(k_{1}, k_{2}\right)$ is classified by $\psi \cdot \phi \cdot k$, and

(d) $\theta \cdot \psi \cdot \phi \cdot t\left(k_{1}, k_{2}\right)$ is classified by $\theta \cdot \psi \cdot \phi \cdot k$.

Then (a) $\Leftrightarrow$ (b) by Lemma 5.2(ii), (b) $\Leftrightarrow$ (c) by Lemma 5.7(ii), and (c) $\Leftrightarrow$ (d) by Lemma 5.4(ii). Hence (ii) is verified.

(i) We have just seen that the function of (i) agrees with the group action of Proposition 3.11(i). It follows that the function of (i) is a group action.

Proposition 1.1 is a reformulated version of Proposition 5.9(i), in that we use an alternative description of the group action. With the Proposition 1.1 approach the action is slightly easier to describe in elementary notation. In particular, we do not need to introduce additional terminology concerning maps, produced via the homotopy extension property.

Proof of Proposition 1.1. Let $\theta \in \operatorname{Aut} G, \phi \in A u t(H), \psi \in M^{0}(\bar{K}, \bar{L})$, and $t\left(k_{1}, k_{2}\right)$ be the Postnikov fibration corresponding to $k \in M^{0}\left(B, M_{\infty}\right)$, We know by Proposition $3.9(\mathrm{v})$ that $\psi \cdot\left(\theta \cdot t\left(k_{1}, k_{2}\right)\right)$ is $1 \mathrm{FHE}$ to $\theta \cdot\left((\psi \circ \bar{\theta}) \cdot t\left(k_{1}, k_{2}\right)\right)$. Applying the fibred exponential law (4.2), it follows that the corresponding based maps, $B \rightarrow M_{\infty}$, i.e. $\psi \cdot(\theta \cdot k)$ and $\theta \cdot((\psi \circ \bar{\theta}) \cdot k)$, are based homotopic. Replacing $\psi$ by $\psi \circ \bar{\theta}^{-1}$ and $k$ 
by $\psi \cdot k$, we obtain:

$$
\left(\psi \circ \bar{\theta}^{-1}\right) \cdot(\theta \cdot(\phi \cdot k)) \simeq^{0} \theta \cdot(\psi \cdot(\phi \cdot k)) .
$$

It follows that the rule

$$
(\langle\theta,[\psi], \phi\rangle,[k]) \mapsto\left[\left(\psi \circ \bar{\theta}^{-1}\right) \cdot(\theta \cdot(\phi \cdot k)]\right.
$$

is an alternative description of group action of Proposition 5.9. It is in this alternative form that the action is described in Proposition 1.1.

Proof of the Basic Classification Theorem 1.2. We see by (ii) of Proposition 5.9 that the bijection

$$
\left[B, M_{\infty}\right]^{0} \rightarrow 1 \mathrm{FHT}(\bar{K} \times \bar{L}: B), \quad[k] \mapsto\left[t\left(k_{1}, k_{2}\right)\right]
$$

(see 4.4) is equivariant relative to the actions of Propositions 5.9 and 3.11. Hence there is an induced bijection between the two orbit sets corresponding to these actions. The result follows via Theorem 3.12.

\section{A Stable Range Version of the FHT Classification Theorem}

We now show that in situations where $\psi$ can be delooped, i.e. where there is a $\chi \in M^{0}(K, L)$ such that $\Omega \chi \simeq^{0} \psi$, then $\langle\theta, \psi, \phi\rangle \cdot k$ is specified using only composition and pointwise addition of functions. This enables us to use such a $\chi$ to give formulae defining both the homotopy $T$ of (5.5) and the corresponding group action on $\left[B, M_{\infty}\right]^{0}$.

Let $\chi: K \rightarrow L$ be a based map, and $t\left(k_{1}, k_{2}\right)$ a Postnikov fibration over $B$ and with distinguished fibre $\bar{K} \times \bar{L}$. We define the map $k_{1} \sqcap p: B \sqcap\left(k_{1}\right) P K \rightarrow K$ by $\left(k_{1} \sqcap p\right)(b, y)=k_{1}(b)=p(y)$, where $(b, y) \in B \sqcap P K$, and

6.1. $\chi \cdot k_{2}=k_{2}+\chi \circ\left(k_{1} \sqcap p\right) \in M\left(B \sqcap\left(k_{1}\right) P K,\left\{b_{0}\right\} \times \bar{K} ; L, e\right)$,

where + denotes pointwise addition relative to $L$.

If $k: B \rightarrow M_{\infty}$ is the based classifying map for $t\left(k_{1}, k_{2}\right)$, then we define $\chi \cdot k \in$ $M^{0}\left(B, M_{\infty}\right)$ by

$$
(\chi \cdot k)(b)=k(b)+c\left(\chi k_{1}(b)\right) .
$$

In this definition $k(b)$ and $c\left(\chi k_{1}(b)\right)$ are both maps $P K \mid k_{1}(b) \rightarrow L$, and the + refers to pointwise addition. The function $\chi \cdot k$ is continuous because it corresponds to $\chi \cdot k_{2}$ via the fibred exponential law (4.2).

If $\psi=\Omega \chi \in M^{0}(\bar{K}, \bar{L})$, the homotopy

6.3. $S:\left(B \sqcap\left(k_{1}\right) P K\right) \times I \rightarrow L, S(b, y, t)=k_{2}(b, y)+\chi y(t)$

meets the specifications of (3.6), i.e. relative to $k_{2}$ and $\psi_{1}$.

Recalling that $\left(B \sqcap\left(k_{1}\right) P K\right) \times I \cong(B \times I) \sqcap\left(k_{1} \circ \pi\right) P K$ by the rule $(b, y, t) \mapsto$ $(b, t, y)$, as explained in the proof of Lemma $5.7(\mathrm{ii})$, we see that $S$ determines a map $(B \times I) \sqcap\left(k_{1} \circ \pi\right) P K \rightarrow L,(b, t, y) \mapsto k_{2}(b, y)+\chi y(t)$. Then (4.2) determines a map 
6.4 .

$$
T: B \times I \rightarrow M_{\infty}, \quad T(b, t)=k(b)+\varepsilon_{b, t},
$$

where $b \in B, t \in I$, and $k(b)$ and $\varepsilon_{b, t}$ are in $M\left(P K \mid k_{1}(b), L ; c(e)\right)$, with $\varepsilon_{b, t}(y)=$ $\chi y(t)$.

Thus $T(b, t)(y)=k(b)(y)+\varepsilon_{b, t}(y)=k_{2}(b, y)+\chi y(t)$. Further, $T$ extends $k$ and $\psi_{2}$.

Hence $T$ meets the requirements of the homotopy of (5.5), and can be used to define $\psi \cdot k$. Thus if $k_{1}(b)=y(1)$

$$
\begin{aligned}
(\psi \cdot k)(b)(y) & =T(b, 1)(y) \\
& =k(b)(y)+\varepsilon_{b, 1}(y) \\
& =k(b)(y)+\chi y(1) \\
& =k(b)(y)+\chi k_{1}(b) .
\end{aligned}
$$

Hence $(\psi \cdot k)(b)=k(b)+c\left(\chi k_{1}(b)\right)=\chi \cdot k(b)$. So if $\psi=\Omega \chi$, then $\psi \cdot k=\chi \cdot k$. It follows, by $(2.9)$, that

6.5. $\psi \in M^{0}(\bar{K}, \bar{L})$ and $\psi \simeq^{0} \Omega \chi \Longrightarrow \psi \cdot k \simeq^{0} \chi \cdot k$.

Let $\theta \in$ Aut $G, \chi \in M^{0}(K, L), \phi \in$ Aut $H$ and $k \in M^{0}\left(B, M_{\infty}\right)$. Then we define

6.6.

$$
\langle\theta, \chi, \phi\rangle \cdot k=\theta \cdot(\chi \cdot(\phi \cdot k)) \in M^{0}\left(B, M_{\infty}\right) .
$$

So it follows by (5.8), (6.5) and (6.6) that

6.7. $\psi \in M^{0}(K, L)$ and $\psi \simeq^{0} \Omega \chi \Longrightarrow\langle\theta, \chi, \phi\rangle \cdot k \simeq^{0}\langle\theta, \psi, \phi\rangle \cdot k$.

We recall the cohomology suspension function [MT, p.139]

$$
H^{n+1}(K ; H) \rightarrow H^{n}(\bar{K} ; H)
$$

which, in homotopy theoretic terms, is the covariant looping function

$$
\Omega_{*}:[K, L]^{0} \rightarrow[\bar{K}, \bar{L}]^{0}, \quad[\chi] \mapsto[\Omega \chi] .
$$

There is also an associated function

$$
\Lambda_{*}: \mathcal{E}(K \times L) \rightarrow \mathcal{E}(\bar{K} \times \bar{L}), \quad \Lambda_{*}(\langle\theta,[\chi], \phi\rangle)=\langle\theta,[\Omega \chi], \phi\rangle .
$$

It is well known that if $n<2 m$, then $\Omega_{*}$ is an isomorphism (see [MT, Prop.4, p.152]), and follows easily that $\Lambda_{*}$ is also an isomorphism.

Proposition 6.8. (i) If $0<m<n<2 m$, then there is a group action:

$$
\alpha: \mathcal{E}(K \times L) \times\left[B, M_{\infty}\right]^{0} \rightarrow\left[B, M_{\infty}\right]^{0}, \quad(\langle\theta,[\chi], \phi\rangle,[k]) \mapsto[\langle\theta, \chi, \phi\rangle \cdot k] .
$$

(ii) If $\beta$ denotes the action of Proposition 5.9(i), then $\alpha=\beta \circ\left(\Lambda_{*} \times 1\right)$.

Proof. (ii) follows by (6.7), and (i) is a consequence of (ii)

Proof of Proposition 1.3. This result is just (i) of Proposition 6.8, but with the formula describing the action spelled out in more detail.

Proof of the Stable Range Classification Theorem 1.4. The two orbit sets $\left[B, M_{\infty}\right]^{0} / \mathcal{E}(\bar{K} \times \bar{L})$ of Theorem 1.2 and $\left[B, M_{\infty}\right]^{0} / \mathcal{E}(K \times L)$ of Theorem 1.4 agree (see Proposition 6.8(ii) and use the fact that $\Lambda^{*}$ is an isomorphism). The result follows immediately from Theorem 1.2. 
Homology, Homotopy and Applications, vol. 8(2), 2006

\section{The Principal Fibration Case}

7.1. Let $X, Y$ and $Z$ be spaces, and $f: X \rightarrow Y$ and $g: Y \rightarrow Z$ be maps. Then we will use the notation

$$
\prec f, g \succ: X \rightarrow Y \times Z \quad \text { for the map } \quad x \mapsto(f(x), g(x)) .
$$

It is standard that principal $\bar{K} \times \bar{L}$-fibrations over $B$ are classified up to equivariant FHE by maps $B \rightarrow K \times L$ (see [B2, Thm.5.8 and Prop.5.9]). Thus $p \times q: P K \times$ $P L \rightarrow K \times L$ is universal amongst such principal fibrations. If $k_{1} \in M^{0}(B, K)$ and $k_{2}^{\wedge} \in M^{0}(B, L)$, then the classifying map

$$
\prec k_{1}, k_{2}^{\wedge} \succ: B \rightarrow K \times L
$$

corresponds to the principal fibration $\prec k_{1}, k_{2}^{\wedge} \succ^{*}(p \times q)$. Interpreted as a Postnikov fibration, this fibration is $t\left(k_{1}, k_{2}^{\wedge} \circ\left(\left(k_{1}\right)^{*} p\right)\right)$, with classifying map

$$
B \rightarrow M_{\infty}, \quad b \mapsto c\left(k_{2}^{\wedge}(b)\right): P K \mid k_{1}(b) \rightarrow L
$$

7.2. It is explained in (M1) of [B1, p.89] that there is a based embedding

$$
j_{\infty}: K \times L \rightarrow M_{\infty}, \quad(x, w) \mapsto c(w): P K \mid x \rightarrow L
$$

where $x \in K$ and $w \in L$, that is fibrewise over $K$.

We now relate $j_{\infty}$ to the universal fibration $t\left(p_{\infty}, e_{\infty}\right)$ of $(4.3)$.

Proposition 7.3 (Properties of $\left.j_{\infty}\right)$. (i) The induced fibration $\left(j_{\infty}\right)^{*} t\left(p_{\infty}, e_{\infty}\right)$ is - to within a canonical homeomorphism of its total space - the principal $\bar{K} \times \bar{L}$-fibration $p \times q: P K \times P L \rightarrow K \times L$

(ii) Then $j_{\infty} \circ \prec k_{1}, k_{2}^{\wedge} \succ$ is the classifying map — in the sense of (4.3) - for the principal fibration $\prec k_{1}, k_{2}^{\wedge} \succ^{*}(p \times q)$.

(iii) Let $\left(k_{1}, k_{2}\right)$ be a Postnikov fibration over $B$ with distinguished fibre $\bar{K} \times \bar{L}$, and associated classifying map $k$. Then $t\left(k_{1}, k_{2}\right)$ is a principal fibration if and only if $k$ factors through $j_{\infty}$, i.e. if and only if there exists a map $f: B \rightarrow K \times L$ such that $j_{\infty} \circ f=k$.

Proof. (i) Viewing the induced fibration as a Postnikov fibration $E_{2} \rightarrow E_{1} \rightarrow K \times$ $L, E_{1}$ is obtained by pulling back $p: P K \rightarrow K$ over $p_{\infty} \circ j_{\infty}$, i.e. over the projection $K \times L \rightarrow K$. Hence the principal $\bar{K}$ - fibration $E_{1} \rightarrow K \times L$ is $p \times 1_{L}: P K \times L \rightarrow$ $K \times L$.

Then $E_{2}$ is obtained by pulling back the path fibration $q: P L \rightarrow L$ over the projection $P K \times L \rightarrow L$. Hence the principal $\bar{L}$ - fibration $E_{2} \rightarrow E_{1}$ may be taken to be the map $1_{P K} \times q: P K \times P L \rightarrow P K \times L$. The result follows.

(ii) This follows from (i) by elementary properties of pullbacks.

(iii) $\Rightarrow$ If $t\left(k_{1}, k_{2}\right)$ is a principal fibration then, for some choice of $f \in M^{0}(B, K \times$ $L), t\left(k_{1}, k_{2}\right)=f^{*}(p \times q)=f^{*} j_{\infty}^{*} t\left(p_{\infty}, e_{\infty}\right)=\left(j_{\infty} \circ f\right)^{*} t\left(p_{\infty}, e_{\infty}\right)$. Now $k_{1}$ and $k_{2}$ determine $k$ uniquely, so $k=j_{\infty} \circ f$, and $k$ factors through $k_{\infty}$. $\Leftarrow t\left(k_{1}, k_{2}\right)=k^{*} t\left(p_{\infty}, e_{\infty}\right)=f^{*} j_{\infty}^{*} t\left(p_{\infty}, e_{\infty}\right)=f^{*}(p \times q)$. So $t\left(k_{1}, k_{2}\right)$ is the pullback of a principal fibration, and hence is itself principal. 
Let $D, W$ and $X$ be pointed spaces and $g: W \rightarrow X$ be a pointed map. Then there is a covariantly induced function $g_{*}:[D, W]^{0} \rightarrow[D, X]^{0}, g_{*}([f])=[g \circ f]$.

Lemma 7.4. Let $D$ have the pointed homotopy type of a pointed $C W$-complex and connectivity greater than or equal to some positive integer $n$. If $g$ induces isomorphisms of homotopy groups in dimensions $>n$, then the induced function $g_{*}:[D, W]^{0} \rightarrow[D, X]^{0}$ is a bijection.

Proof. This is a slight variation of the corresponding result where $g$ is a weak homotopy equivalence [S, Cor.7.6.23].

Proposition 7.5. Let $1<m<n<2 m$, and $B$ have connectivity greater than $n-$ $m+1$. Then the induced function $\left(j_{\infty}\right)_{*}:[B, K \times L]^{0} \rightarrow\left[B, M_{\infty}\right]^{0}$ is a bijection.

Proof. $\pi_{i}\left(M^{0}(\bar{K}, L ; c(e))\right) \approx\left[\bar{K}, \Omega^{i} L\right]^{0}$ (utilizing repeated applications of the exponential law for cg-spaces $) \approx[\bar{K}, K(H, n+1-i)]^{0} \approx H^{n+1-i}(\bar{K} ; H)$. This is 0 if $n+1-i<m$, i.e. if $i>n-m+1$.

If $N$ is a CW-complex, with distinguished point $n_{0}$, then the based map $v=$ $v(N): M(N, L ; c(e)) \rightarrow L$, that evaluates at $n_{0}$, is a fibration (the proof follows easily via the expononential law for cg-spaces [V, Thm.3.6]).

Now $\bar{K}$ has the homotopy type of a CW-complex $N$, so the analogue of $v$ for $\bar{K}$, i.e. $v(\bar{K}): M(\bar{K}, L ; c(e)) \rightarrow L$, is a quasi-fibration with essentially the same exact homotopy sequence as $v(N)$. Further, $v(\bar{K})$ has a section $j_{0}: L \rightarrow M(\bar{K}, L ; c(e))$, with $j_{0}(w)(y)=w$, where $y \in \bar{K}$ and $w \in L$, and its distinguished fibre is $\left.M^{0}(\bar{K}, L ; c(e))\right)$.

Inserting known zero values of $\pi_{i}\left(M^{0}(\bar{K}, L ; c(e))\right.$ into the exact homotopy sequence for $v(\bar{K})$, we see that $\pi_{i}(v): \pi_{i}(M(\bar{K}, L ; c(e))) \rightarrow \pi_{i}(L)$ is an isomorphism for $i>n-m+1$. Now $\pi_{i}\left(j_{0}\right)$ is a right inverse to $\pi_{i}(v)$, so $\pi_{i}\left(j_{0}\right)$ is an isomorphism for the same range of values.

The map $j_{\infty}$ (see 7.2) is a fibrewise map from the projection $K \times L \rightarrow K$ to the fibration $p_{\infty}: M_{\infty} \rightarrow K$. Its restriction to the distinguished fibre $\{e\} \times L=L$ is $j_{0}$. It follows, via the associated exact homotopy ladder and the five-lemma, that $\pi_{i}\left(j_{\infty}\right): \pi_{i}(K \times L) \rightarrow \pi_{i}\left(M_{\infty}\right)$ is an isomorphism for $i>n-m+1$. Hence $\left(j_{\infty}\right)_{*}$ is a bijection for the specified range of values of $i$ (Lemma 7.4).

Proof of Proposition 1.5. There is a group action

$$
\mathcal{E}(K \times L) \times[B, K \times L]^{0} \rightarrow[B, K \times L]^{0}
$$

defined by composition. The action of Proposition 1.5 is just the cohomology interpretation of that action.

Proof of the Stable Range Classification Theorem 1.6. (i) We have to verify that the Hurewicz fibration case is equivalent to the principal fibration case, and this follows immediately from (2.10), Proposition 7.3(ii) and Proposition 7.5.

(ii) The function $\left(j_{\infty}\right)_{*}$ is equivariant relative to the group actions described in the proof of Proposition 1.5 and in Proposition 6.8(i). Then Proposition 7.5 ensures 
that the function induced by $j_{\infty}$, i.e.

$$
[B, K \times L]^{0} / \mathcal{E}(K \times L) \rightarrow\left[B, M_{\infty}\right]^{0} / \mathcal{E}(K \times L,)
$$

is a bijection.

The result follows if we compose the last bijection with that of Theorem 1.4, and reinterpret $[B, K \times L]^{0} / \mathcal{E}(K \times L)$ in terms of cohomology.

(iii) The orbit of $(\kappa, \lambda) \in H^{m+1}(B ; G) \oplus H^{n+1}(B ; H)$ corresponds to the orbit of the homotopy class of $\langle u, v\rangle$ in $[B, K \times L]^{0}$. This in turn corresponds via Proposition 7.5 to the orbit of the homotopy class of $j_{\infty} \circ\langle u, v\rangle$ in $\left[B, M_{\infty}\right]^{0}$, which then corresponds via Theorem 1.4 to the fibrewise homotopy type of $\left(j_{\infty} \circ\langle u, v\rangle\right)^{*} t\left(p_{\infty}, e_{\infty}\right)$. We know via Proposition 7.3(ii) that this is the orbit of $\langle u, v\rangle^{*}(p \times q)$ as required.

\section{Examples}

Example 8.1. If $H=0$, then $K(H, n)$ can be a one point space, and $M_{\infty} \cong K$ (see (M2) of $[\mathrm{B} 1, \mathrm{p} .89])$. Hence

$$
\left[B, M_{\infty}\right]^{0} / \mathcal{E}(K(G, m) \times K(0, n)) \approx H^{m+1}(B ; G) / \text { Aut } G,
$$

so we have retrieved the classification theorem for fibrations whose fibres have a single non-zero homotopy group.

Example 8.2. If $G=0$, then $K(G, m)$ can be a one point space, and $M_{\infty} \cong L$ (see (M2) of [B1, p.89]). Hence

$$
\left[B, M_{\infty}\right]^{0} / \mathcal{E}(K(0, m) \times K(H, n)) \approx H^{m+1}(B ; H) / \text { Aut } H,
$$

and we have again retrieved the classification theorem for fibrations whose fibres have a single non-zero homotopy group.

Example 8.3. We use Theorem 1.6 to compute the set $\operatorname{FHT}(K(G, m) \times K(H, n)$ : $\left.S^{t} \times S^{u}\right)$, where $m, n, t$ and $u$ are positive integers with $0<m<n<\min (2 m$, $m+t-2)$.

(i) If $t<u$, the above set of fibrewise homotopy types is in bijective correspondence with:

(a) $(G /$ Aut $G) \times(H /$ Aut $H)$ if $m=t-1$ and $n=u-1$.

(b) $G$ /Aut $G$

if $m=t-1<n<2 t-3$, but $n \neq u-1$,

or $m=u-1<n<t+u-3$, or

$m=t+u-1<n<2 t+u-3$.

(c) $H /$ Aut $H$

if $\frac{t-1}{2}<m<t-1=n$, or $\max \left(\frac{u-1}{2}, u-t+1\right)<m<u-1=n$,

but $m \neq t-1$, or

$u+1<m<t+u-1=n$.

(d) 0

if $m, n \notin\{t-1, u-1, t+u-1\}$. 
(ii) If $t=u$, the above set of fibrewise homotopy types is in bijective correspondence with:
(a) $(G \oplus G) /$ Aut $G$
if $m=t-1<n<2 t-3$.
(b) $G /$ Aut $G$
if $m=2 t-1<n<3 t-3$.
(c) $(H \oplus H) /$ Aut $H$
if $\frac{t-1}{2}<m<t-1=n$.
(d) $H$ /Aut $H$
if $t+1<m<2 t-1=n$.
(e) 0
if $m, n \notin\{t-1,2 t-1\}$.

(iii) The cases where the classifying set has a single element, i.e. (i)(d) and (ii)(e), are independent of the $n<\min (2 m, m+t-2)$ assumption.

The actions of $G$ and $H$ on $G \oplus G$ and $H \oplus H$, respectively, are the diagonal actions. Thus, in the former case, the rule is defined by $(\theta, u, v) \mapsto(\theta(u), \theta(v))$, where $\theta \in \operatorname{Aut}(G)$, and $u, v \in G$.

\section{9. $\quad$ Proofs for Section 3}

Proof of Lemma 3.2. (i) If $\phi$ and $\phi^{\prime} \in$ Aut $H$, then

$$
\begin{aligned}
\phi^{\prime} \cdot\left(\phi \cdot t\left(k_{1}, k_{2}\right)\right) & =\phi^{\prime} \cdot\left(t\left(k_{1}, \phi \circ k_{2}\right)\right) \\
& =t\left(k_{1}, \phi^{\prime} \circ \phi \circ k_{2}\right) \\
& =t\left(k_{1}, \overline{\left.\left(\phi^{\prime} \circ \phi\right) \circ k_{2}\right)}\right. \\
& =\left(\phi^{\prime} \circ \phi\right) \cdot t\left(k_{1}, k_{2}\right) .
\end{aligned}
$$

(ii) The fibrewise map $\Phi(\phi)$ is a homeomorphism, since it has an inverse $\Phi\left(\phi^{-1}\right)$, and so is certainly a FHE. Also the restriction of $\Phi(\phi)$ to the distinguished fibre $\left\{b_{0}\right\} \times \bar{K} \times \bar{L}$, or equivalently $\bar{K} \times \bar{L}$, is $1 \times \bar{\phi}: \bar{K} \times \bar{L} \rightarrow \bar{K} \times \bar{L}$, as required.

Proof of Lemma 3.4. This argument is parallel to that of Lemma 3.2, and uses routine properties of pullbacks. The verification of (i) depends on the facts that postcomposition with $\underline{\theta}$ and precomposition by $\theta_{\wedge}^{-1}$ both depend covariantly on $\theta$. The fibrewise map $\Theta(\theta)$ of (ii) is a homeomorphism, since it has inverse $\Theta\left(\theta^{-1}\right)$. The restriction of $\Theta(\theta)$ to the distinguished fibre $\left\{b_{0}\right\} \times \bar{K} \times \bar{L}=\bar{K} \times \bar{L}$ is $\theta \times 1: \bar{K} \times$ $\bar{L} \rightarrow \bar{K} \times \bar{L}$, as required.

Proof of Lemma 3.5. The results of (i) follow via the exponential law for cg-spaces. Thus $\psi_{1}$ may be determined from $\psi$ by applying that law once, and $\psi_{2}$ derives from $\psi_{1}$ by applying it again. The results of (ii) are obtained if we apply similar procedures to a based homotopy $\bar{K} \times I \rightarrow \bar{L}$.

Proof of Lemma 3.8. (i) Let $\psi$ and $\psi^{\prime} \in M^{0}(\bar{K}, \bar{L})$. Defining $\psi^{\prime} \cdot\left(\psi \cdot k_{2}\right)$ involves applying the cofibration property twice to $k_{2}$, first using $\psi_{1}$ and then $\psi_{1}^{\prime}$. 
However if we define $\psi_{1}+\left(\psi^{\prime}\right)_{1}: \bar{K} \times I \rightarrow L$ by

$$
\left(\psi_{1}+\left(\psi^{\prime}\right)_{1}\right)(y, t)= \begin{cases}\psi_{1}(y, 2 t) & t \leqslant 1 / 2 \\ \left(\psi^{\prime}\right)_{1}(y, 2 t-1) & t \geqslant 1 / 2\end{cases}
$$

then a single application of the cofibration property using $k_{2}$ and $\psi_{1}+\left(\psi^{\prime}\right)_{1}=$ $\left(\psi+\psi^{\prime}\right)_{1}$ yields a member of the same homotopy class $\left(E_{1},\left\{b_{0}\right\} \times \bar{K}\right) \rightarrow(L,\{e\})$ (see $(2.9))$. Hence $\psi^{\prime} \cdot\left(\psi \cdot t\left(k_{1}, k_{2}\right)\right)$ and $\left(\psi+\psi^{\prime}\right) \cdot t\left(k_{1}, k_{2}\right)$ have the same 1FHT, and it follows that we are dealing with a group action.

(ii)(a) We consider a Postnikov fibration $t\left(k_{1}, k_{2}\right)$, with associated principal fibrations $p_{1}: E_{1} \rightarrow B$ and $p_{2}: E_{2} \rightarrow E_{1}$, and apply the argument of (2.10), with $r: Z \rightarrow B$ taken as $q: P L \rightarrow L, A=E_{1}, H=S\left(k_{1}, k_{2}, \psi\right)$ of $(3.6), f=k_{2}$ and $g=$ $\psi \cdot k_{2}$. There is a specific procedure for computing liftings over path fibrations (see [S, Cor.2.8.8]) and so, after following through the details, we obtain a homotopy $R: E_{2} \times I \rightarrow P L$ with restriction $R_{1}: E_{2} \rightarrow P L$, where

$$
R_{1}(b, y, z)(t)= \begin{cases}z(2 t) & t \leqslant 1 / 2 \\ S(b, y, 2 t-1) & t \geqslant 1 / 2\end{cases}
$$

$b \in B, y \in P K, z \in P L$, and $(b, y, z) \in E_{2}$. We must also consider the Postnikov fibration $t\left(k_{1}, \psi \cdot k_{2}\right)$, with principal fibrations $p_{1}: E_{1} \rightarrow B$ as before, and $p_{2}^{\psi}: E_{2}^{\psi} \rightarrow$ $E_{1}$, where $E_{2}^{\psi}=E_{1} \sqcap\left(\psi \cdot k_{2}\right) P L$ and $p_{2}^{\psi}=\left(\psi \cdot k_{2}\right)^{*}(q)$.

Then, using $\Psi=\Psi(\psi)$ to denote the corresponding FHE $h$, we have

$$
\Psi: E_{2} \rightarrow E_{2}^{\psi}, \quad(b, y, z) \mapsto\left(b, y, R_{1}(b, y, z)\right) .
$$

(b) Now $t\left(k_{1}, k_{2}\right)^{-1}\left\{b_{0}\right\}=\left\{b_{0}\right\} \times \bar{K} \times \bar{L}=t\left(k_{1}, \psi \cdot k_{2}\right)^{-1}\left\{b_{0}\right\}$ (see (3.7)), hence the restriction over $\left\{b_{0}\right\}$ of the FHE $\Psi$ is (essentially) a self-homotopy equivalence of $\bar{K} \times \bar{L} \rightarrow \bar{K} \times \bar{L}$.

(c) The restriction of $R_{1}$ over $\left\{b_{0}\right\}$ is essentially

$$
R_{1} \mid\left(\left\{b_{0}\right\} \times \bar{K} \times \bar{L}\right): \bar{K} \times \bar{L} \rightarrow \bar{L}, \quad(y, z) \mapsto R_{1}\left(b_{0}, y, z\right)=z+S\left(b_{0}, y,-\right),
$$

where $\left\{b_{0}\right\} \times \bar{K} \times \bar{L}$ is equated with $\bar{K} \times \bar{L}$, and + is the operation on $\bar{L}$.

Now $S: E_{1} \times I \rightarrow L$ extends $\psi_{1}:\left\{b_{0}\right\} \times \bar{K} \times I=\bar{K} \times I \rightarrow L$, so $S\left(b_{0}, y,-\right)=$ $\psi_{1}(y,-) \in \bar{L}$. Hence $\psi_{1}(y,-)(t)=\psi_{1}(y, t)=\psi(y)(t)$, where $y \in \bar{K}, t \in I$, and so $\psi_{1}(y,-)=\psi(y)$. So we have shown that if $y \in \bar{K}$ and $z \in \bar{L}$, then $R_{1}(y, z)=z+$ $\psi(y)$.

(d) Hence $\Psi: E_{1} \rightarrow E_{1}^{\psi}$ restricted over $b_{0} \in B$ is

$$
\bar{K} \times \bar{L} \rightarrow \bar{K} \times \bar{L}, \quad(y, z) \mapsto(y, z+\psi(y)) .
$$

Now $\langle 1, \psi, 1\rangle \times\langle y, z\rangle=\langle y, \psi(y)+z\rangle$, so the homotopy commutativity of the operation on $\bar{L}$ ensures that $\Psi$ is a $(1, \psi, 1)$-FHE.

Proof of Lemma 3.9. Parts (i), (ii) and (iii) are contained in parts (i) of Lemmas $3.2,3.4$, and 3.8 , respectively. The proofs of (i) and (iii) are spelled out in the proofs of Lemmas 3.2 and 3.8. We verify the most difficult of the remaining three cases, i.e. $(\mathrm{v})$, and leave the others to the reader.

We notice that $\psi \cdot\left(k_{2} \circ\left(1 \sqcap \theta_{\wedge}^{-1}\right)\right)$ and $\left((\psi \circ \bar{\theta}) \cdot k_{2}\right) \circ\left(1 \sqcap \theta_{\wedge}^{-1}\right)$ are both terminating maps of homotopies that extend the same map, i.e. both are restrictions 
to $\left(B \sqcap\left(\underline{\theta} \circ k_{1}\right) P K\right) \times\{1\}$ of homotopies $\left(B \sqcap\left(\underline{\theta} \circ k_{1}\right) P K\right) \times I \rightarrow L$, both of which extend both $k_{2} \circ\left(1 \sqcap \theta_{\wedge}^{-1}\right):\left(B \sqcap\left(\underline{\theta} \circ k_{1}\right) P K\right) \times\{0\} \rightarrow L$ and $\psi_{1}:\left\{b_{0}\right\} \times \bar{K} \times I \rightarrow$ $L$. It follows, via (2.9), that these homotopy extensions are homotopic via a homotopy that extends the constant homotopy $\left\{b_{0}\right\} \times \bar{K} \times I \rightarrow L$ with value $e \in L$.

Each of these terminating maps induces the " $E_{2} \rightarrow E_{1}$ " fibration of a specific Postnikov fibration, in both cases with $E_{1}=B \sqcap\left(\underline{\theta} \circ k_{1}\right) P K$. It follows by the relative version of (2.10) that there is an FHE, between these fibrations, that restricts to the identity over $\left\{b_{0}\right\} \times \bar{K}$. Hence this FHE is a 1 FHE between the corresponding Postnikov fibrations " $E_{2} \rightarrow B$ ".

So $\psi \cdot\left(\theta \cdot t\left(k_{1}, k_{2}\right)\right)=\psi \cdot t\left(\underline{\theta} \circ k_{1}, k_{2} \circ\left(1 \sqcap \theta_{\wedge}^{-1}\right)\right)$ which is $1 \mathrm{FHE}$ to $\left.t\left(\underline{\theta} \circ k_{1},\left((\psi \circ \bar{\theta}) \cdot k_{2}\right) \circ\left(1 \sqcap \theta_{\wedge}^{-1}\right)\right)\right)=\theta \cdot t\left(k_{1},(\psi \circ \bar{\theta}) \cdot k_{2}\right)=\theta \cdot\left((\psi \circ \bar{\theta}) \cdot t\left(k_{1}, k_{2}\right)\right)$.

\section{Acknowledgements}

The author would like to thank NSERC Canada for its financial support, and the University of Wales in Bangor for its hospitality during the summers of 2003 and 2005, both of which contributed to the development of this work.

\section{References}

Ba. H. J. Baues, Obstruction Theory, Lecture Notes in Math. 628, Springer-Verlag, Berlin 1977.

B1. Peter I. Booth, Fibrations with Product of Eilenberg-MacLane Space Fibres I, Contemp. Math. 274 (2001), 79-104.

B2. Peter I. Booth, Fibrations and Classifying Spaces: Overview and the Classical Examples, Cahiers de Topologie et Géométrie Différentielle Catégorique $\mathbf{4 1}$ (2000), 162-206.

B3. Peter I. Booth, Mapping Spaces and Homotopy Theory, Quaestiones Mathematicae 27 (2004), 415-430.

K. Donald W. Kahn, Induced Maps for Postnikov Systems, Trans. Amer. Math. Soc. 107 (1963), 432-450.

MT. Robert E. Mosher and Martin C. Tangora, Cohomology Operations and Applications in Homotopy Theory, Harper and Row, New York, 1968.

R. John W. Rutter, Groups of Self-Homotopy Equivalences of Induced Spaces, Comment. Math. Helv. 45 (1970), 236-255.

S. Edwin H. Spanier, Algebraic Topology, McGraw-Hill, New York, 1966.

St. N. E. Steenrod, Cohomology Operations and Obstructions to Extending Continuous Functions, Mimeographed notes, University, 1957; reprinted in Advances in Mathematics 8 (1972), 371-416.

V. Rainer M. Vogt, Convenient Categories of Topological Spaces for Homotopy Theory, Arch. Math. 22 (1971), 545-555.

W. George W. Whitehead, Elements of Homotopy Theory, Springer-Verlag, Berlin, 1978. 
Peter I. Booth pbooth@math.mun.ca

Department of Mathematics and Statistics

Memorial University

Elizabeth Avenue

St John's NL A1C5S7

Canada

This article is available at http://intlpress.com/HHA/v8/n2/a8 